\title{
Relación entre lumbalgia y sobrepeso/ obesidad: dos problemas de salud pública
}

\author{
Javier Ernesto Matta Ibarra - Víctor Elías Arrieta Maríab - Juan Carlos \\ Andrade Rodríguez ${ }^{c}$ - Daniel Marcos Uruchi Limachi ${ }^{d}$ - Jonathan Alberto Lara \\ Taveras $^{\mathrm{e}}$ - Saith del Carmen Trouchón Jiméne ${ }^{\mathrm{f}}$
}

\begin{abstract}
Resumen: La lumbalgia y el sobrepeso-obesidad son patologías que se han constituido en tema de interés en salud pública, dado el incremento de incidencia y prevalencia de estas enfermedades en la población mundial. Investigaciones al respecto han sido realizadas especialmente en población anglosajona. Este artículo presenta un estudio analítico de corte transversal, en el que se incluyeron 100 pacientes escogidos con un muestreo por conveniencia, con diagnóstico de lumbalgia, y atendidos en la consulta externa institucional de Ortopedia-Columna del Hospital Militar Central, durante el periodo de abril-junio de 2015. Se analizaron variables sociodemográficas y relacionadas con severidad del dolor, sobrepeso-obesidad e imbalance muscular, variables que se consideraban factores asociados a la presentación y severidad de lumbalgia. De los pacientes analizados, el $52 \%$ fueron mujeres y el $48 \%$, hombres. Se encontró que las mujeres con porcentaje de grasa corporal elevado tienen un riesgo mayor de padecer dolor lumbar severo, y los hombres con porcentaje de grasa corporal bajo tienen menos riesgo de presentar esta afección. El índice de masa corporal (IMC) para este estudio no pudo predecir la intensidad de dolor lumbar; sin embargo, se logró observar que ser hombre mayor de 50 años es un factor de riesgo para presentar lumbalgia severa.
\end{abstract}

Palabras clave: dolor de la región lumbar; índice de masa corporal; sobrepeso; obesidad

Fecha de recepción: 23 de agosto de 2018 Fecha de aprobación: 20 de diciembre de 2018

a Ortopedista y traumatólogo. Director del Programa de Especialización en Cirugía de Columna, Universidad Militar Nueva Granada. Jefe de la Clínica de Columna, Hospital Militar Central.

Correo electrónico: jematta@yahoo.es

b Ortopedista y traumatólogo. Docente del Programa de Especialización en Cirugía de Columna, Universidad Militar Nueva Granada. Jefe de la Cínica de Pelvis y Acetábulo, Hospital Militar Central.

c Ortopedista y traumatólogo. Especialista en formación en el Programa de Especialización en Cirugía de Columna, Universidad Militar Nueva Granada. Hospital Militar Central.

d Ortopedista y traumatólogo. Universidad Mayor de San Andrés, La Paz Bolivia. Fellow AOSpine, Latinoamérica. Hospital Militar Central.

e Médico residente nivel IV Ortopedia y Traumatología. Hospital Docente Universitario Dr. Darío Contreras, Santo Domingo República Dominicana. Programa Graduado Observador en Cirugía de Columna, Hospital Militar Central.

f Médico residente nivel III Ortopedia y Traumatología. Universidad Militar Nueva Granada, Hospital Militar Central 
Cómo citar: Matta Ibarra JE, Arrieta María VE, Andrade Rodríguez JC, Uruchi Limachi DM, Lara Taveras JA, Trouchón Jiménez S del C. Relación entre lumbalgia y sobrepeso/ obesidad: dos problemas de salud pública. Revista Med.27(1):53-60. Disponible en: https://revistas.unimilitar.edu.co/index.php/rmed/article/ view/4755

\title{
Relationship Between Low Back Pain and Overweight/Obesity: Two Public Health Problems
}

\begin{abstract}
Low back pain and overweight/obesity have become a topic of interest in public health, given the increased incidence and prevalence of these pathologies in the world population. Research in this regard has been carried out, especially in the Anglo-Saxon population. This article presents a cross-sectional analytical study, which included 100 patients chosen by convenience sampling, with a diagnosis of low back pain and attending the institutional Orthopedics-Column outpatient clinic of the Hospital Militar Central during April-June 2015. Sociodemographic variables related to pain severity, overweight/obesity, and muscular imbalance were analyzed, as they were considered factors associated with the occurrence and severity of low back pain. Of the patients analyzed, $52 \%$ were women and $48 \%$ men. It was found that women with high fat percentage have a higher risk of severe low back pain, while men with low fat percentage have a lower risk of having this condition. Body mass index (BMI) for this study could not predict the intensity of low back pain; however, it was observed that being a man older than 50 is a risk factor for severe low back pain.
\end{abstract}

Keywords: Lower back pain; body mass index; overweight; obesity

\section{Relação entre lumbago e sobrepeso-obesidade: dois problemas de saúde pública}

Resumo: O lumbago e o sobrepeso-obesidade são patologias que se constituem em tema de interesse em saúde pública, tendo em vista o aumento de incidência e a prevalência dessas doenças na população em todo o mundo. Pesquisas sobre isso têm sido realizadas, em especial, com população anglo-saxã. Este artigo apresenta um estudo analítico, de corte transversal, do qual participaram 100 pacientes selecionados por conveniência, com diagnóstico de lumbago, e atendidos em consulta institucional de Ortopedia-Coluna do Hospital Militar Central, entre abril e junho de 2015. Foram analisadas variáveis sociodemográficas e relacionadas com severidade da dor, sobrepeso-obesidade e desequilíbrio muscular, variáveis consideradas fatores associados com a apresentação e a severidade de lumbago. Dos pacientes analisados, $52 \%$ eram mulheres e $48 \%$, homens. Foi verificado que as mulheres com porcentagem de gordura elevada têm um risco maior de padecer dor lombar severa, e os homens com porcentagem de gordura baixa têm menos risco de apresentar essa condição. O índice de massa corporal para este estudo não pode predizer a intensidade de dor lombar; contudo, pôde-se observar que ser homem com mais de 50 anos é um fator de risco para apresentar lumbago grave.

Palavras-chave: dor na região lombar; índice de massa corporal; sobrepeso; obesidade 


\section{Introducción}

La lumbalgia es considerada un problema común que se puede experimentar en algún momento de la vida, y el dolor uno de los principales motivos de consulta en los centros médicos, debido a que este limita de forma importante la vida laboral de los individuos (1).

Esta patología se caracteriza por dolor, tensión muscular o rigidez localizada entre el borde inferior de la última costilla y por encima de los pliegues glúteos inferiores. Esta sintomatología puede o no estar acompañada por dolor irradiado a los miembros inferiores (ciática) (2),

Los datos de prevalencia son interesantes: a nivel mundial se encuentran entre el $1,0 \%$ y el $58,1 \%$ (3) y para Latinoamérica se calculan cifras de $10,5 \%$ (4). Por otra parte, la incidencia anual de dolor lumbar oscila entre $6,3 \%$ y $15,4 \%$, con lo que se puede inferir que los episodios de dolor pueden ser recurrentes en el tiempo y esto aumenta las cifras hasta un $36 \%$ (3).

Es importante mencionar que la lumbalgia es una enfermedad que no solo impacta al individuo en sus diferentes aspectos, sino que además influye de manera significativa en los sistemas de salud, dado que constantemente se incurre en gastos derivados de los aumentos de tiempo de incapacidad, así como en indemnizaciones. A nivel mundial esta enfermedad ocupa el primer lugar en términos de discapacidad y el sexto en enfermedad general (4). En Australia el costo de la enfermedad representó una carga económica masiva y un problema de salud importante que llegó a los \$9,2 billones AUD (5), mientras que para el Reino Unido esta patología le costó al sistema de salud aproximadamente $£ 11.000$ millones en 2000 (3). En Serbia, el costo de tratamiento aproximado fue de $€ 200,40$ por paciente por año, y los mayores volúmenes de costos fueron debido a las visitas a especialistas en instituciones de atención primaria de salud $(€ 9,39)(6)$.

Es preciso mencionar, además, que para México durante 2007 se otorgaron 27,8 millones de días de incapacidad, con un promedio de 12 días por persona, lo que en peso mexicano corresponde a un gasto de $\$ 5000$ millones (7).

Información reportada por la guía europea para el manejo del dolor crónico afirma que alrededor del $11 \%$ al $12 \%$ de la población tiene discapacidad por dolor lumbar (8), lo que evidencia un panorama de lo que sucede en el mundo y nos lleva a cuestionar la magnitud del problema en Colombia.

De acuerdo con un estudio realizado en Cali, a medida que la edad aumenta también incrementa la lumbalgia, las incapacidades el tratamiento y, por ende, los costos de esta (9); sin embargo, no está claro cuál es la cifra exacta de su impacto económico. Además, se evidenció un aumento proporcional entre la lumbalgia, la incapacidad, el tratamiento y su costo. Por ejemplo, durante 2014 el Hospital Militar Central atendió 3920 pacientes que consultaron por lumbalgia, que corresponden al $14 \%$ de todos los pacientes atendidos por ortopedia.

Cuando se habla del origen de la enfermedad, solo del $5 \%$ al $15 \%$ de los casos tienen una causa específica, por ejemplo, una fractura por osteoporosis, neoplasias o infección; sin embargo, para el 85-95\% de los casos restantes no hay una causa específica clara del dolor lumbar, pero se conocen factores de riesgo que podrían influir en el inicio o la recurrencia de la lumbalgia, como factores ambientales, personales y psicológicos, algunos de los cuales podrían ser modificables (3).

Dentro de los factores modificables se encuentra el peso corporal, que al aumentar genera sobrepeso y obesidad, siendo esta una enfermedad que afectó a más de un tercio de la población mundial para 2015 (10), y causó alrededor de 3,4 millones de muertes durante 2010 (11). Lo anterior refleja que si esta enfermedad continúa en aumento, para 2030 el $38 \%$ de la población sufrirá de sobrepeso y el $20 \%$ tendrá obesidad (10).

De acuerdo con el informe de indicadores básicos generado por la Organización Panamericana de la Salud (ops), para 2017 en las Américas se contaba con una población total de $1.006 .000 \mathrm{mi}$ llones de habitantes, de los cuales 49.066 millones corresponden a la población colombiana, de la que se ha calculado una tasa de sobrepeso y obesidad de $54,2 \%$ para hombres y 58,0 \% para mujeres (12).

Con el aumento anual de estas tasas los gastos médicos se incrementan por las hospitalizaciones, los medicamentos y las comorbilidades asociadas a esta enfermedad. El gasto por año para un hombre obeso es de US $\$ 1,152$ mientras que para 
las mujeres este valor se duplica a US \$3,613 (valores de 2005 para Estados Unidos); de acuerdo con esto, se calculó que se gastan aproximadamente US $\$ 190$ billones por año, es decir, el $21 \%$ de los gastos en salud. De igual modo, en Europa para 2008 este costo estaba alrededor de $€ 10,4$ billones anuales (10). En conclusión, la obesidad es uno de los principales problemas de salud pública en el mundo y está categorizada como una pandemia $(13,14)$.

Se cree que las causas del aumento de peso en la población están relacionadas con los cambios en la alimentación y el pobre estilo de vida, lo que da como consecuencia el aumento del Índice de Masa Corporal (IMC); se considera que a mayor IMC se intensifica el riesgo de contraer enfermedades crónicas, y a esto se suman trastornos musculoesqueléticos como el dolor lumbar $(13,14)$.

Teniendo en cuenta lo anterior, se evidencia una relación importante entre el sobrepeso y la obesidad como un factor de riesgo para la lumbalgia. Algunos estudios han revelado que el sobrepeso y la obesidad acrecienta la prevalencia del dolor lumbar (15-17), lo que lleva a generar carga biomecánica anormal, pérdida de masa muscular en los miembros inferiores, incremento de los niveles de inflamación sistémica y los niveles de artritis (18). Esto hace que más personas consulten por dolor lumbar, y eleva proporcionalmente la prevalencia de esta enfermedad; además, produce un alto número de discapacidades temporales o indefinidas, lo que hace que sea un serio problema de salud pública por tratar.

\section{Materiales y métodos}

Se realizó un estudio analítico de corte trasversal, utilizando un muestreo por conveniencia, que incluyó datos de una muestra total de 100 pacientes con diagnóstico de lumbalgia, atendidos en la consulta externa de columna del Área de Ortopedia y Traumatología del Hospital Militar Central, en el periodo abril-junio de 2015. Se excluyeron los pacientes con enfermedad articular inflamatoria, antecedentes de fusión lumbar a cualquier nivel, patología neuromuscular y antecedentes de patología traumática, tumoral o deformidades. La información clínica de los pacientes se obtuvo mediante la revisión de las historias clínicas con el fin de extraer toda la información relacionada con las variables de interés para el estudio, como sociodemográficas y aquellas relacionadas con severidad del dolor, sobrepeso-obesidad e imbalance muscular (perímetro abdominal aumentado-limitación funcional de flexión lumbar por retracción de isquiotibiales); variables que se consideran asociadas a la presentación y severidad de lumbalgia. Para determinar el IMC se utilizó la fórmula convencional dividiendo los kilogramos de peso por el cuadrado de la estatura en metros; el porcentaje de grasa corporal se calculó con el pliegue cutáneo subescapular utilizando un plicómetro, que es una pinza que atrapa la piel y mide el grosor en milímetros. El perímetro abdominal se midió con una cinta métrica que da el resultado en centímetros. Para evaluar el dolor en los sujetos se empleó la escala visual análoga (EVA) representada en una línea de $10 \mathrm{~cm}$, que gradúa el dolor desde la ausencia de este hasta un dolor intenso. Finalmente, el análisis por imágenes de columna lumbosacra se efectuó mediante radiografías simples y en algunos casos, según conveniencia, con tomografía computarizada o resonancia magnética.

Si estos estudios eran normales, es decir no se detectaba en ellos alguna patología específica, la lumbalgia se consideraba mecánica. Los datos se procesaron con el paquete estadístico spss versión 19. Para las variables numéricas se usaron estadísticos descriptivos como promedios, desviación estándar, valor mínimo y valor máximo; las variables cualitativas se analizaron mediante frecuencias y porcentajes. Se utilizaron tablas de $2 \times 2$ y la prueba estadística de chi cuadrado para el análisis bivariado de variables categóricas y modelos de regresión logística para identificar factores de asociación con lumbalgia.

\section{Resultados}

En el grupo de 100 pacientes incluidos en el estudio hubo una distribución por género casi homogénea, y entre los dos grupos se encontró un $52 \%$ de mujeres y un $48 \%$ de hombres. La media de edad fue de 55 años, lo que mostró que el $63 \%$ de los pacientes era mayor de 50 años. El peso medio fue de $74,8 \mathrm{~kg}$, y una talla media de $162,7 \mathrm{~cm}$. Con respecto al IMC, el promedio fue de 28,2 (Tabla 1 ). 
Tabla 1. Frecuencias de edad, peso, talla e IMC

\begin{tabular}{|l|l|l|l|l|} 
& Promedio & Des Std. & Mínimo & Máximo \\
\hline Edad & 55 & 19,8 & 20 & 86 \\
\hline Peso & 74,8 & 14,8 & 45 & 143 \\
\hline Talla & 162,7 & 11,2 & 140 & 194 \\
\hline IMC & 28,2 & 4,15 & 19,5 & 38,6 \\
\hline
\end{tabular}

Fuente: elaboración propia.

En la distribución del IMC los pacientes presentaron sobrepeso en un $51 \%$, seguido de $22 \%$ que evidencia obesidad grado I (tabla 2). La intensidad media del dolor lumbar de acuerdo con la Eva fue de 7 (DS $1,89)$, y la mayor frecuencia de grado de dolor fue 8 (28\%), que corresponde a dolor severo (figura 1 ).

Tabla 2. Distribución del IMC .

\begin{tabular}{|l|l|l|}
\hline IMC & Frecuencia & $\%$ \\
\hline Normal & 17 & 17,00 \\
\hline Sobrepeso & 51 & 51,00 \\
\hline Obesidad I & 22 & 22,00 \\
\hline Obesidad II & 10 & 10,00 \\
\hline
\end{tabular}

Fuente: elaboración propia.

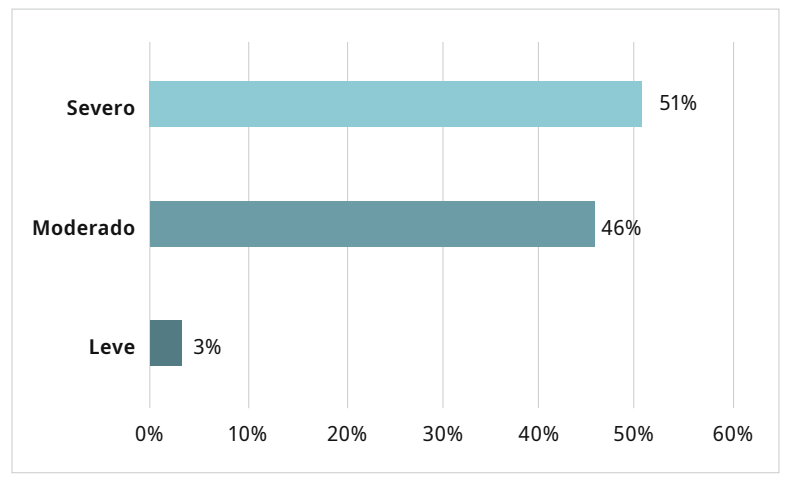

Figura 1. Intensidad del dolor

Fuente: elaboración propia.

En cuanto a la distribución del patrón del dolor, el dolor lumbar aislado fue el más frecuente puesto que se presentó en el $50 \%$ de los pacientes, seguido del dolor lumbar radicular, en el $41 \%$ de los casos (figura 2).

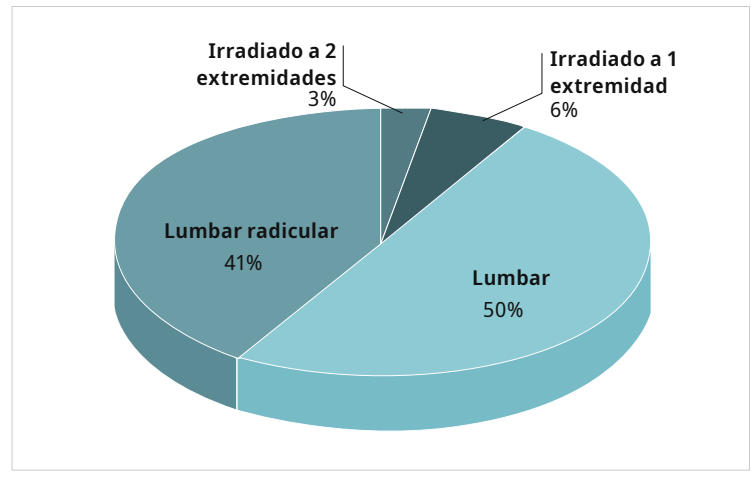

Figura 2. Distribución del patrón de dolor

Fuente: elaboración propia.

Frente al porcentaje de grasa corporal general de los pacientes, este se encontró elevado en un $71 \%$ de los casos: $33 \%$ en hombres y $38 \%$ en mujeres. La media del porcentaje de grasa en pliegues corporales fue de 20,2 y el porcentaje de grasa más frecuente 20 en 10 pacientes (10\%). El perímetro corporal abdominal se observó elevado en un $68 \%$, característica que se presenta más alta en las mujeres, con $44 \%$, y en los hombres con $24 \%$. Finalmente, para el perímetro troncantérico, la media de los pacientes fue de 99,6 cm (tabla 3).

Tabla 3. Valores de grasa en pliegues y perímetros corporales

\begin{tabular}{|l|l|l|l|l|} 
& Promedio & Des. Std. & Mínimo & Máximo \\
\hline $\begin{array}{l}\text { Grasa en } \\
\text { pliegues }\end{array}$ & 20,2 & 8,3 & 5 & 55 \\
\hline $\begin{array}{l}\text { Perímetro } \\
\text { torácico }\end{array}$ & 95,5 & 11,5 & 57 & 133 \\
\hline $\begin{array}{l}\text { Perímetro } \\
\text { abdominal }\end{array}$ & 93,2 & 12,8 & 58 & 140 \\
\hline $\begin{array}{l}\text { Perímetro } \\
\text { trocantérico }\end{array}$ & 99,6 & 13,1 & 50 & 132 \\
\hline
\end{tabular}

Fuente: elaboración propia.

Al realizar un análisis bivariado general se observó que existía una distribución homogénea de dolor de leve a severo entre géneros; el dolor es mayor en pacientes con IMC en sobrepeso con una tendencia a la obesidad, y se evidencia una disminución de los rangos de flexión lumbar en pacientes con dolor severo. La localización de dolor es mayor en región lumbar y el perímetro abdominal 
tiene una distribución similar entre rangos de dolor de leve a severo (tabla 4).

Tabla 4. Análisis bivariado general

\begin{tabular}{|l|l|l|} 
& $\begin{array}{l}\text { Dolor leve a } \\
\text { moderado }\end{array}$ & Severo \\
\hline Género & & \\
\hline Masculino & $23(46,9 \%)$ & $25(49 \%)$ \\
\hline Femenino & $26(53 \%)$ & $26(50,9 \%)$ \\
\hline IMc & & \\
\hline Sobrepeso & $25(51 \%)$ & $26(50,9 \%)$ \\
\hline Obesidad & $15(30,6 \%)$ & $17(33,3 \%)$ \\
\hline Flexión lumbar & & \\
\hline III & $31(63,2 \%)$ & $40(78,4 \%)$ \\
\hline Localización del dolor & & \\
\hline Lumbar & $24(48,9 \%)$ & $26(50,9 \%)$ \\
\hline Perímetro abdominal & & $34(66,6 \%)$ \\
\hline Elevado & $34(69,3 \%)$ & \\
\hline
\end{tabular}

Fuente: elaboración propia.

En cuanto al análisis por genero se encontró que el $78,3 \%$ de los hombres que presentaron dolor leve a moderado tenían IMC con sobrepeso u obesidad, el $60 \%$ que presentaba intensidad severa de lumbalgia tenía porcentaje de grasa alto, el 21,7\% de los pacientes con intensidad de lumbalgia leve a moderada podían realizar flexión lumbar grado IV, mientras que solo el $12 \%$ con lumbalgia severa alcanzó este mismo grado de flexión.

Para el grupo de mujeres se observó que aquellas que tenían lumbalgia severa presentaron porcentaje de grasa elevado en un $80,8 \%$ de los casos. Aquellas con intensidad de dolor lumbar leve a moderado tienen mayor grado de flexión que aquellas con lumbalgia severa $(23,7 \%$ contra $7,69 \%$ para flexión grado IV). Todas las mujeres tenían perímetro abdominal aumentado sin importar la intensidad del dolor.

Al aplicar el coeficiente de correlación de Pearson para el análisis bivariado general y por género no se encontraron relaciones estadísticamente significativas. En la tabla 5, se presentan los resultados del análisis multivariado con los Odds Ratio, donde se puede observar que ser hombre mayor de 50 años implica ocho veces más riesgo de lumbalgia severa. En ese mismo grupo, aquellos con un porcentaje de grasa bajo tienen menor riesgo de dolor lumbar severo. Las mujeres con porcentaje de grasa elevado presentan un riesgo cuatro veces mayor de padecer lumbalgia severa.

Tabla 5. Análisis multivariado

\begin{tabular}{|c|c|c|c|}
\hline & $\begin{array}{l}\text { Dolor } \\
\text { lumbar } \\
\text { severo }\end{array}$ & OR & $P>Z$ \\
\hline Mujeres & $\begin{array}{l}\text { Porcentaje } \\
\text { grasa alto }\end{array}$ & $\begin{array}{c}4,28 \text { (IC } 1,01- \\
18,1)\end{array}$ & 0,048 \\
\hline \multirow[t]{2}{*}{ Hombres } & $\begin{array}{l}\text { Mayores de } \\
50 \text { años }\end{array}$ & $\begin{array}{c}7,81 \text { (IC } 1,75- \\
34,9)\end{array}$ & 0,007 \\
\hline & $\begin{array}{l}\text { Porcentaje } \\
\text { grasa bajo }\end{array}$ & $\begin{array}{c}0,18 \text { (IC } 0,04- \\
0,81)\end{array}$ & 0,026 \\
\hline
\end{tabular}

Fuente: elaboración propia.

En cuanto a los diagnósticos desde el punto de vista clínico-imagenológico, predominarón el canal lumbar estrecho con un $28 \%$ de casos, hernia discal con un $25 \%$ y en $22 \%$ no se detectaron patologías específicas por lo que fueron clasificados como "lumbalgia mecánica" (tabla 6).

Tabla 6. Diagnóstico clínico - imagenológico

\begin{tabular}{|l|c|}
\hline Diagnóstico clínico-imagenológico & $\%$ \\
\hline Canal lumbar estrecho & 28 \\
\hline Espondilolistesis ístmica & 8 \\
\hline Hernia discal & 25 \\
\hline Listesis degenerativa & 11 \\
\hline Lumbalgia mecánica & 22 \\
\hline Escoliosis degenerativa & 1 \\
\hline Otros diagnósticos & 5 \\
\hline
\end{tabular}

Fuente: elaboración propia.

\section{Discusión}

Este estudio buscó investigar la posible asociación entre lumbalgia y sobrepeso-obesidad, además de buscar otras relaciones como edad, genero, perímetro abdominal, IMC y porcentaje de grasa subescapular. Se pudo evaluar que el dolor de espalda en región lumbar no tuvo relaciones significativas con el género, pero la frecuencia de consulta fue 
similar tanto para hombres, como para mujeres. Por otra parte, dentro de los resultados se encontró que la localización más frecuente del dolor fue en la región lumbar y lumbar radicular, y su intensidad se concentró en los niveles de moderado a severo. Estos hallazgos son compatibles con estudios relacionados que caracterizan los factores sociodemograficos de población con dolor lumbar y obesidad $(19,20)$.

En cuanto al IMC respecto a la lumbalgia, se observó en el grupo de estudio que los pacientes que consultaron por dolor tenían sobrepeso, y al realizar el análisis entre el IMC y el dolor lumbar no se encontró una relación entre estas dos variables; sin embargo, otros estudios muestran que a mayor IMC se da una asociación entre dolor lumbar tanto en hombres, como mujeres (21).

En este estudio no se halló una relación entre dolor y perímetros antropométricos; empero, resultados de otras investigaciones muestran que la distribución regional de la adiposidad está asociada al dolor lumbar crónico (22). El análisis teniendo en cuenta el género determina, en este estudio, que las mujeres con sobrepeso o con un perímetro abdominal aumentado tienen una probabilidad significativamente mayor de dolor lumbar (23). No obstante, otra literatura no apoya esta relación, y concluye que no hay razones lo suficientemente fuertes para determinar que el IMC, el porcentaje de masa grasa y masa alrededor de las caderas tienen que ver con la prevalencia de dolor lumbar (24). Por el contrario, para el grupo de los hombres esta relación no fue significativa (23), pero sí se encontró una asociación en la cual ser hombre mayor de 50 años es un factor de riesgo para presentar lumbalgia (25).

Así, de acuerdo con el estudio, tanto el porcentaje de grasa escapular como el perímetro abdominal son significativamente altos para los hombres y para las mujeres, lo que incluye cambios en la marcha y presencia de estrés articular.

Como conclusión se puede decir que, aunque no se encuentran relaciones significativas entre el IMC y el dolor lumbar, el sobrepeso y la obesidad se diagnosticaron en el $83 \%$ de los pacientes, lo que hace necesario recomendar cambios en el estilo de vida para el control de este factor de riesgo en enfermedades crónicas, por ejemplo, de tipo cardiovascular o diabetes, lo cual impactaría de manera positiva en la calidad de vida de los sujetos; además, debe hacerse hincapié en medidas preventivas en los jóvenes y en todas las etapas del curso de la vida, para disminuir dichos índices.

Asimismo, se observó dentro de la literatura que no hay datos concluyentes entre la relación de sobrepeso-obesidad y dolor lumbar, lo que concuerda con los datos de este estudio. Para futuras investigaciones de este tipo se puede sugerir incluir un número mayor de sujetos. Una limitante en esta investigación fue la no inclusión de variables como las relacionadas con componentes psico-somáticos o laborales, estrato socioeconómico, nivel educativo y comportamientos de estilo de vida no saludable, por ejemplo, el consumo de alcohol y cigarrillo.

\section{Conflictos de interés}

Los autores declaran no tener conflicto de interés en la realización de este trabajo.

\section{Financiación}

Esta investigación fue financiada directamente por los investigadores.

\section{Referencias}

[1] Ganesan S, Acharya AS, Chauhan R, Acharya S. Prevalence and Risk Factors for Low Back Pain in 1,355 Young Adults: A Cross-Sectional Study. Asian Spine J. 2017 ag.;11(4):610-17.

[2] Koes BW, van Tulder MW, Thomas S. Diagnosis and treatment of low back pain. BMJ. 2006 jun. 17; 332(7555): 1430-4.

[3] Hoy D, Brooks P, Blyth F, Buchbinder R. The Epidemiology of low back pain. Best Pract. Res Clin Rheumatol. 2010 dic.;24(6):769-81.

[4] Vos T, Allen C, Arora M, Barber RM, Bhutta ZA, Brown A, et al. (2016). Global, regional, and national incidence, prevalence, and years lived with disability for 310 diseases and injuries, 1990-2015: a systematic analysis for the Global Burden of Disease Study 2015. Lancet. 2016 oct. 8;388(10053):1545-1602. DOI: https:// doi.org/10.1016/s0140-6736(16)31678-6.

[5] Walker BF, Muller R, Grant WD. Low Back Pain in Australian Adults: The Economic Burden. Asia Pac. 
J Public Health. 2003;15(2):79.87. DOI: https:/doi. org/10.1177/101053950301500202.

[6] Radoičić MJ, Božović BD, Ilić KD, Jankovic SM, Anđelković JZ, Kostić MJ. Pharmacoeconomic Aspects of Low Back Pain Treatment: Cost of Illness Study in the Republic of Serbia. Acta Med Port. 2019 abr. 30;32(4):272-8. DOI: http://dx.doi.org/10.20344/ amp. 10910 .

[7] Covarrubias-Gómez A. Lumbalgia: un problema de salud pública. Rev Mex Anest. 2010;33(S1):S106-S9.

[8] Airaksinen O, Brox JI, Cedraschi C, Hildebrandt J, Klaber-Moffett J, Kovacs F, et al. Chapter 4. European guidelines for the management of chronic nonspecific low back pain. Eur Spine J. 2006 mar.;15(S2):S192-300.

[9] Arce-Eslava SL, García-Lozano EJ, Parra-González E, Cruz-Libreros AM. Costos por dolor lumbar en una EPS en Cali, Colombia. Rev Col Salud Ocup. 2013 jun.;3(2):22-25.

[10] Hruby A, Hu FB. The Epidemiology of Obesity. A big picture. Pharmacoeconomics. 2015 jul.;33(7):673-89.

[11] Pengpid S, Peltzer K. Association between risk factors and overweight and obesity among adults in poblation-based samples from 31 countries. Obes Res Clin Pract. 2017 mar.-Abr.;11(2):158-66.

[12] Organización Panamericana de la Salud (OPS), Organización Mundial de la Salud (OMS). Situación de Salud en las Américas: Indicadores Básicos 2017 [Internet]. Washington, D.C.: Estados Unidos; 2017. Disponible en: http://www.paho.org/data/index.php/es/ indicadores.html

[13] Dario AB, Ferreira ML, Refshauge K, Sánchez-Romera JF, Luque-Suarez A, Hopper JL. Are obesity and body fat distribution associated with low back pain in women? A population-based study of 1128 Spanish twins. Eur Spine J. 2016 abr.;25(4):1188-95.

[14] Córdova JA, Barriguete JA, Rivera ME, Lee MG, Mancha C. Sobrepeso y obesidad. Situación actual y perspectivas. Act Méd Grupo Ángeles. 2010 oct.dic.;8(4):202-7.

[15] Shiri R, Karppinen J, Leino-Arjas P, Solovieva S, Viikari-Juntura E. The association between obesity and low back pain: a metaanalysis. Am. J. Epidemiol. 2010 en. 15;171(2):135-54.
[16] Leboeuf-Y de C, Kyvik KO, Bruun NH. Low back pain and lifestyle. Part II-Obesity. Information from a population-based sample of 29,424 twin subjects. Spine (Phila Pa 1976). 1999 abr. 15;24(8):779-83.

[17] Heuch I, Heuch I, Hagen K, Zwart JA. Body mass index as a risk factor for developing chronic low back pain: a follow-up in the Nord-Trøndelag Health Study. Spine (Phila Pa 1976). 2013 en. 15;38(2):133-9.

[18] Wilson Zingg R, Kendall R. Obesity, Vascular Disease, and Lumbar Disk Degeneration: Associations of Comorbidities in Low Back Pain. PM R. 2017 abr.;9(4):398-402.

[19] Llaguno López R, Martínez T, Janett M, Álvarez Licona N. Caracterización sociodemográfica de los pacientes que acudieron a un servicio de rehabilitación de tercer nivel de atención por lumbalgia. Rev Sanidad Militar. 2018 ag. 15;72(1):5-9.

[20] Brooks C, Siegler JC, Marshall PW. Relative abdominal adiposity is associated with chronic low back pain: a preliminary explorative study. BMC Public Health. 2016 ag. $2 ; 16: 700$

[21] Han TS, Schouten JS, Lean ME, Seidell JC. The prevalence of low back pain and associations with body fatness, fat distribution and hight. Int J Obes Relat Metab Disord. 1997 jul.;21(7):600-7.

[22] Dario AB, Ferreira ML, Refshauge K, Sánchez-Romera JF, Luque-Suarez A, Hopper JL, et al. Are obesity and body fat distribution associated with low back pain in women? A population-based study of 1128 Spanish twins. Eur Spine J. 2016 abr.;25(4):1188-95.

[23] Hashimoto Y, Matsudaira K, Sawada SS, Gando Y, Kawakami R, Kinugawa C, et al. Obesity and low back pain: A retrospective cohort study of Japanese males. J Phys Ther Sci. 2017 jun.;29(6):978-83.

[24] Frilander H, Solovieva S, Mutanen P, Pihlajamäki H, Heliövaara M, Viikari-Juntura E. Role of overweight and obesity in low back disorders among men: a longitudinal study with a life course approach. BMJ Open. 2015 ag. 21;5(8): e007805.

[25] Sadler SG, Spink MJ, Ho A, De Jonge XJ, Chuter VH. Restriction in lateral bending range of motion, lumbar lordosis and hamstring flexibility predicts the development of low back pain: a systematic review of prospective cohort studies. BMC Musculoskelet Disord. 2017 may. 5;18(1):179. 